\title{
Calidad del empleo en grupos socialmente vulnerables en México. El caso de los adultos mayores
}

\section{Quality of employment in socially vulnerable groups in Mexico. The case of the elderly}

\author{
Lucio Flores-PAYAN* \\ Iván Alejandro Salas-Durazo*
}

\begin{abstract}
This paper analyzes the quality of employment of the elderly over a period of twelve years using alternative and multidimensional methodologies, such as the theory of fuzzy sets. The results found distinguish different conditions in terms of quality of employment in relation to levels of education, access to health services, and working conditions. Moreover, it is evident that the analyzed age group tends to increasingly live in more precarious conditions showing the partial response from the public policies associated to the attention of this group.
\end{abstract}

Keywords: socially vulnerable groups, elderly people, quality of employment, fuzzy logic models.

\section{Resumen}

En la presente investigación se analiza la calidad del empleo de los adultos mayores durante un periodo de doce ańos, para lo cual se utilizaron metodologías alternativas y multidimensionales, como la teoría de los conjuntos difusos. Los resultados encontrados distinguen condiciones diferenciadas en términos de la calidad del empleo con relación a los niveles de educación, el acceso a servicios de salud y las condiciones laborales. Asimismo, se evidencia que el grupo etario analizado se encuentra en condiciones cada vez más precarias lo cual muestra la parcial respuesta proveniente de las políticas públicas asociadas a la atención de este grupo.

Palabras Clave: grupos socialmente vulnerables, adultos mayores, calidad del empleo, modelos de lógica difusa.

\footnotetext{
*Universidad de Guadalajara, correos-e: florespayan@hotmail.com, ivan.salas@cucea.udg.mx
} 


\section{Introducción}

En la literatura es reiterativo el argumento de que la población de adultos mayores se encuentra en condiciones de vulnerabilidad por diferentes factores, mayormente acotados a la reducción de las capacidades físicas y mentales. Sin embargo, no se detallan las condiciones estructurales como la pobreza, la falta de oportunidades educativas y la generalizada informalidad laboral a la que se enfrentó dicha generación, las cuales provocan que este grupo continúe trabajando en condiciones precarias en detrimento de su calidad de vida.

En la década de los cincuenta, $88 \%$ de la población se encontraba en pobreza patrimonial, $73 \%$ en pobreza de capacidades y $61 \%$ en pobreza alimentaria. Esto permite deducir que en la actualidad la población de adultos mayores proviene de una época en donde se presentaron altos niveles de pobreza, falta de posibilidades educativas y pocas libertades para la generación de capacidades y construcción de redes sociales de apoyo, lo cual propició niveles elevados de analfabetismo, desnutrición y desinterés social (Székely, 2005). Prueba de lo anterior es que de acuerdo con el Consejo Nacional de Evaluación (Coneval), 45.90\% de las personas con 60 años y más se encuentra en situación de pobreza a la vez que $71.90 \%$ cuenta con al menos una carencia social. ${ }^{1}$ Cabe destacar que esta situación no es exclusiva de este grupo ya que el propio Coneval reporta que en $2014,46.10 \%$ de la población se encontraba en estado de pobreza.

Consecuentemente, en la actualidad las problemáticas a las que se enfrentan los adultos mayores se agudizan, lo cual los coloca en una situación de vulnerabilidad. De acuerdo con la Secretaría de Desarrollo Social (Sedesol, 2010) el nivel de ingreso y las bajas tasas de ahorro han propiciado el incremento de adultos mayores en situación de pobreza, además de enfrentar problemas de salud, desempleo, baja cobertura en pensiones y en protección social. Complementariamente y, sin ser el argumento central del presente trabajo, se puede mencionar que la acción gubernamental se ha visto reflejada a través de la implementación de estrategias para frenar el crecimiento poblacional, así como políticas públicas de salud y protección social que han impactado positivamente en la esperanza de vida de las personas.

Paralelamente, es evidente un proceso de transición y transformación demográfica que se caracteriza por el envejecimiento de su población. Este cambio fue clave para modificar el panorama de la atención a la vejez y que resultó a su vez en la incorporación del fenómeno como pro-

\footnotetext{
${ }^{1}$ Las carencias sociales determinadas por dicho organismo son rezago educativo, nulo acceso a los servicios de salud, a la seguridad social, a los servicios básicos en la vivienda y a la alimentación; así como a la falta de calidad en los espacios de la vivienda.
} 
blema público en la agenda gubernamental. Esto facultó el diseño e implementación de políticas públicas para atender a este tipo de grupos socialmente vulnerables.

El presente escrito tiene como principal objetivo analizar la calidad del empleo de los adultos mayores en México. Se parte de la hipótesis de que la combinación de pobreza, el nulo acceso a oportunidades educativas y la generalizada informalidad laboral han conducido a que este grupo etario continúe participando en la dinámica laboral dada su incapacidad estructural para acceder a una pensión. En consecuencia, los empleos a los que acceden presentan alta precariedad teniendo como consecuencia una afectación en la calidad de vida.

\section{El concepto de vulnerabilidad social en los adultos mayores}

La vulnerabilidad de individuos o grupos sociales se relaciona con las condiciones sociales, culturales y económicas que determinan su calidad de vida, es decir, ésta es un elemento base que se ve afectado por las consecuencias de pertenecer a los llamados grupos vulnerable. En este sentido, el concepto de calidad de vida es amplio, complejo y multifactorial, ya que se relaciona con el grado de satisfacción, bienestar y seguridad que las personas sienten o perciben de sí mismas o del lugar donde viven (Navarro y González-Ramírez, 2010). Dado el carácter multidimensional de dicha conceptualización, han sido varias las formas de abordarla y diversos los métodos de análisis que se han utilizado en su medición; sin embargo, para fines del presente trabajo se destaca su trascendencia por ser parte de la cadena de elementos que determinan las características de los grupos socialmente vulnerables, es decir, mientras mayor vulnerabilidad presente un grupo, mayor detrimento presenta su calidad de vida.

La vulnerabilidad social se identifica como la propensión que tienen individuos, familias o grupos sociales a estar sometidos a eventos y procesos que atenten contra su capacidad de subsistencia derivado de acontecimientos ajenos a su control. Esto implica la consideración de las condiciones del entorno social, de manera que la condición de vulnerabilidad limita el acceso a mayores niveles de bienestar y al ejercicio de los derechos ciudadanos (Golovanevsky, 2007).

Es así que el concepto de vulnerabilidad cobra varios matices, entre ellos pueden distinguirse las corrientes conceptuales que relacionan directamente a las externalidades como uno de los principales elementos que propician e incrementan la vulnerabilidad. Por lo que su atención se vuelve central en la acción gubernamental (Sojo, 2004; Ziccardi, 2000). Complementariamente, se le distingue como un fenómeno multicausal y 
de afectación integral (Busso, 2002; Pizarro, 2001). Para Ziccardi (2000) ésta es evidente en grupos como mujeres, niños, adultos mayores e indígenas, los cuales requieren atención a través de la implementación de estrategias gubernamentales -mediante políticas públicas y programas específicos- con la finalidad de corregir los factores que generan daño a estos grupos a la vez de subsanar las perturbaciones sociales generadas.

Por su parte, Pizarro (2001) asume a la vulnerabilidad social como un proceso multidimensional que comprende desde la base material hasta aspectos como la inseguridad y la sensación de indefensión, pasando por la asociación de la precariedad laboral, la carencia de seguridad social, así como el debilitamiento de la participación en asuntos comunes y públicos. Busso (2002) argumenta que la vulnerabilidad es compleja y multicausal: se configura por varias dimensiones analíticas e incluye aspectos de individuos y hogares, así como características económicas, políticas, culturales y ambientales de la sociedad.

En la revisión del estado del arte con relación al concepto de vulnerabilidad es imposible no referir su vínculo con las condiciones de pobreza, marginación social y exclusión. Villa y Rodríguez (2002) agregan a la discusión la disponibilidad de recursos y las estrategias para enfrentar las afectaciones, señalando que pueden surgir desde el interior de los propios grupos o con apoyo externo mayormente gubernamental. En este sentido, puede distinguirse una idea general sobre los elementos que involucran la vulnerabilidad social de individuos y grupos socialmente vulnerables que a manera de interés del presente trabajo se encuentra el grupo de los adultos mayores.

Para el caso de los adultos mayores, la vulnerabilidad se hace evidente en diferentes aspectos tales como la falta de oportunidades laborales, ingresos insuficientes, precarios sistemas de protección social, sobredemanda de servicios de salud y discriminación social y familiar. En la figura 1 se muestran algunos de los elementos que generan vulnerabilidad en este grupo.

Se destaca que la precariedad salarial, si bien no es la única variable, es una de las que más impactan en la vulnerabilidad de los adultos mayores, ya que los niveles bajos de ingreso desencadenan otros factores como la incapacidad de ahorro, la insuficiencia para atender los denominados gastos de bolsillo de padecimientos o las enfermedades asociadas a la edad. Complementariamente, se asocia la informalidad laboral que inevitablemente conduce a la exclusión de servicios de salud y capacidad para acceder a una pensión en detrimento de la calidad de vida. Lo anterior, genera un círculo vicioso de marginación y pobreza. En ese sentido, y como se mencionará más adelante, la acción gubernamental se ha en- 


\section{Figura 1}

\section{Factores que generan vulnerabilidad en los adultos mayores}

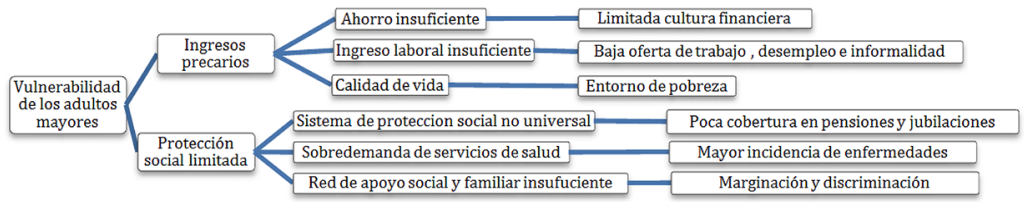

Fuente: elaboración propia a partir de Sedesol (2010).

focado en paliar esta situación a través de programas compensatorios de carácter asistencial.

La vulnerabilidad social, en su carácter multidimensional, facilita entender de una mejor forma los problemas sociales como la pobreza y la exclusión social; asociado el primero a privaciones materiales en función de los bajos ingresos y, el segundo, al debilitamiento de los vínculos entre individuos o grupos con el resto de la comunidad.

De manera integradora estas aproximaciones al concepto de vulnerabilidad social dan lugar a pensar que dicho fenómeno es de carácter polisémico y que para su análisis se requiere de múltiples enfoques metodológicos, o mejor aún, de metodologías y herramientas que faculten la interpretación compleja y multidimensional.

\section{Caracterización de los adultos mayores ocupados en México}

En México, la pirámide poblacional presenta la mayor representación en los grupos de jóvenes y los adultos jóvenes, ya que de acuerdo con la Encuesta Intercensal del Inegi, en 2015, los habitantes menores de 30 años representan $53.07 \%$ del total de población; mientras que el grupo de mayores de 60 años corresponde a $10.40 \%$ del total. Complementariamente, con base en cifras del Inegi en 2016, la expectativa de vida era de 77.8 ańos para las mujeres y 72.6 años para los hombres.

En términos laborales y a partir de los Indicadores Estratégicos del Inegi, durante el primer trimestre de 2016 estaban ocupadas 50,778,629 personas de una población total de 112,803,321, lo cual representa $41.68 \%$. En correspondencia con la distribución poblacional, la masa laboral está mayormente compuesta por adultos jóvenes y medios. De manera que para el mismo periodo, la población ocupada con 60 ańos y más era de 4,591,611, la cual representa 9.04 por ciento. 
En lo que respecta a las características generales del empleo, los salarios del grupo de adultos mayores es aproximadamente $25 \%$, menor que el promedio nacional, aunque en promedio trabajan ocho horas menos por semana. Al margen de lo anterior, su jornada laboral es de tiempo completo tal como se ilustra en la gráfica 1 .

\section{Gráfica 1}

Salario y duración de la jornada laboral promedio en México 2016
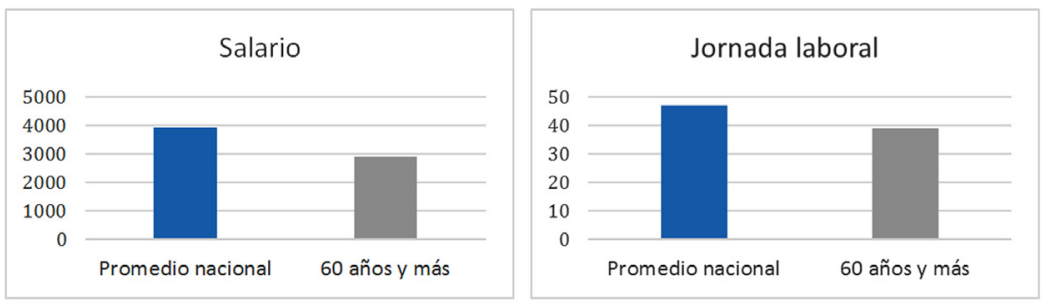

Fuente: elaboración propia a partir de los microdatos de la Encuesta Nacional de Ocupación y Empleo (Inegi, 2016).

En términos de distribución espacial, los adultos mayores ocupados están distribuidos mayormente en la zona centro occidente, centro sur y pacífico sur, como se ilustra en el mapa 1. En ese sentido, Ciudad de México, Estado de México, Veracruz, Jalisco, Michoacán y Puebla concentran $46.16 \%$ del total de ocupados en esta cohorte.

El presente escrito se centra en el grupo de ocupados dentro de la cohorte de edad de 66 a 70 años, considerando que de acuerdo con la Ley del Seguro Social, la edad planteada para solicitar la pensión por vejez es de 65 años. De manera que el análisis se concentrará en dimensionar la calidad del empleo de los ocupados que se encuentran en una condición distinta a la expectativa de una trayectoria laboral promedio en términos normativos.

Las razones por las cuales las personas dentro de esta cohorte se encuentren laborando pueden ser diversas; sin embargo, como punto de partida podríamos suponer que la generalizada informalidad laboral en nuestro país repercute negativamente en la capacidad de retiro de las personas, específicamente en términos del cumplimiento del número mínimo de semanas cotizadas. ${ }^{2}$

Con base en las cifras de la Encuesta Intercensal 2015, el grupo quinquenal de 65 a 69 años estaba conformado por 2,987,316 personas de las

${ }^{2}$ Con base en el artículo 162 de la Ley de Seguro Social en complemento con los 65 años de edad el trabajador que solicite pensión por vejez requiere haber cotizado al menos 1250 semanas. 


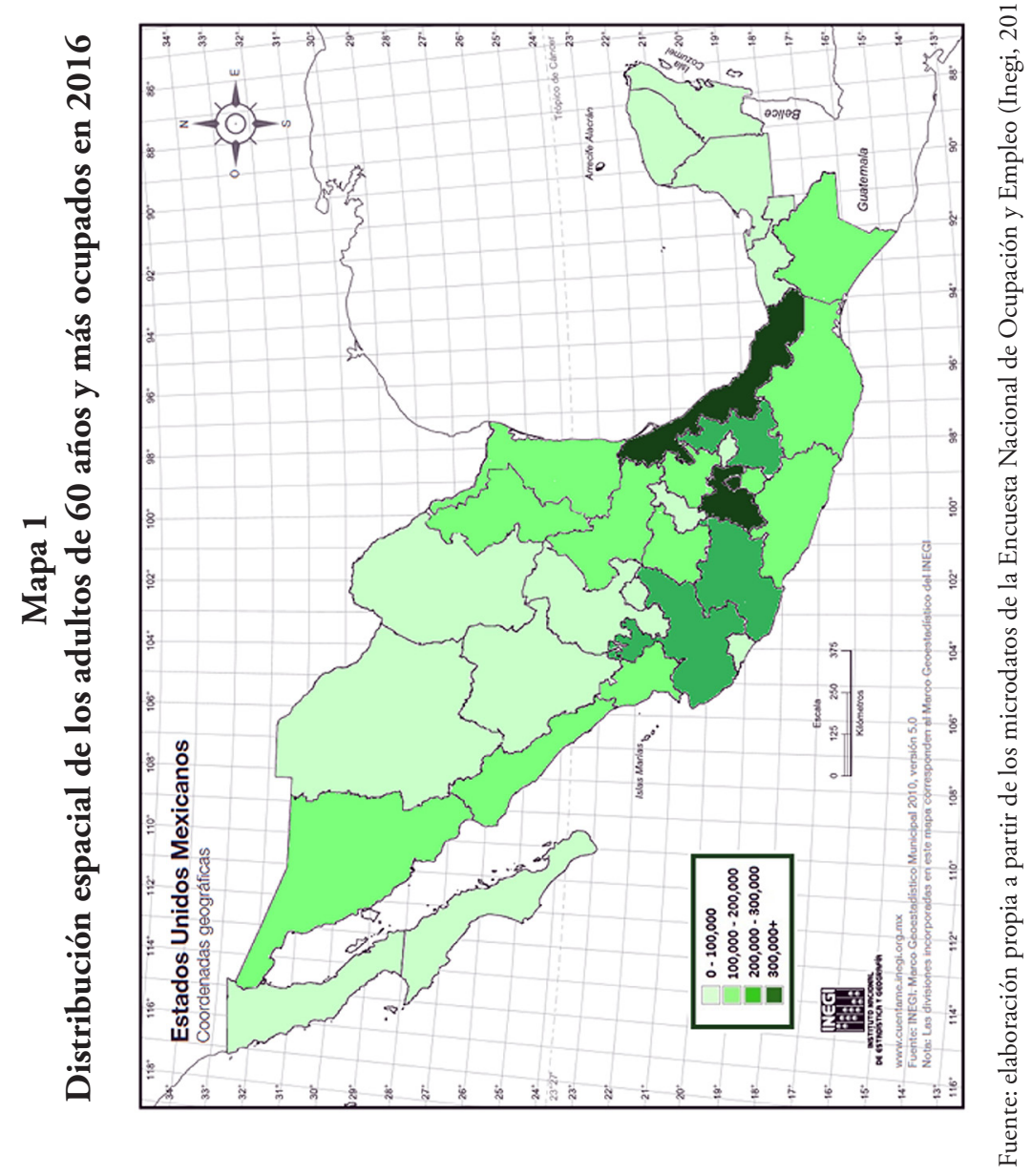


cuales $30.53 \%$ estaba clasificada dentro del grupo de la población económicamente activa donde $96.90 \%$ de este grupo estaba ocupada; es decir, 883,754 trabajadores. Como se señaló anteriormente, para el presente análisis, se emplearon los microdatos de la ENOE en el periodo comprendido del 2005 al 2016. Como referencia, al primer trimestre de 2016 se desprende que para el grupo de edad de 66 a 70 ańos se reportaron $1,023,971$ ocupados.

Como se había anticipado en las secciones anteriores, las condiciones laborales en las que participan los adultos mayores son distintas -y desventajosas- en comparación con las del trabajador promedio. En la gráfica 2 se muestra la distribución por tipo de empleo.

\section{Gráfica 2}

Proporción de ocupados por tipo de empleo en 2016

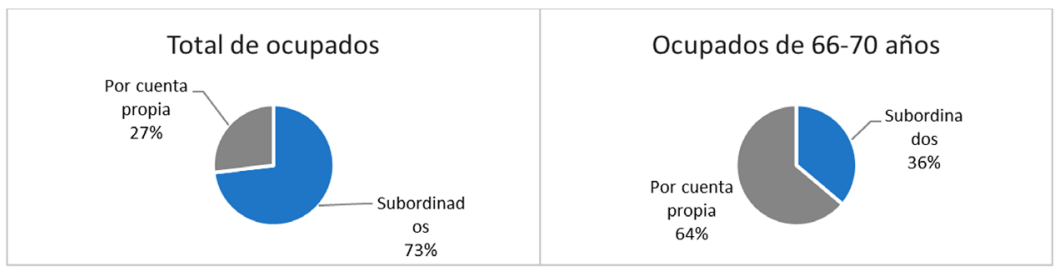

Fuente: elaboración propia con base en los microdatos de la Encuesta Nacional de Ocupación y Empleo (Inegi, 2016).

Como se observa, la manera como se desarrolla el trabajo es distinta para este grupo: el trabajo por cuenta propia en nuestro país tiene una connotación hacia la informalidad, por lo que podríamos anticipar la vulnerabilidad que supone esta condición. Por otra parte, las teorías del desarrollo sitúan a la educación superior como uno de los elementos clave para generar crecimiento. En la gráfica 3 se muestra el nivel de preparación académica del grupo analizado en una serie de tiempo del 2005 al 2016.

Como se ilustra, el nivel académico de los ocupados dentro de la cohorte 66-70 es mayormente menor a licenciatura. Esto es evidente debido a que la cobertura de este nivel para dichas generaciones era prácticamente nulo.

En un sentido general, el trabajo en la tercera edad supone serias desventajas con respecto al grueso de la masa laboral, ya que además de los datos señalados están implícitos otros factores no tan fácilmente cuantificables, asociados al estado de salud, a las capacidades físicas y mentales, al grado de alfabetización digital, a las competencias laborales y de discri- 


\section{Gráfica 3}

Distribución de los ocupados en la cohorte de edad de 66-70 años por tipo de empleo y educación

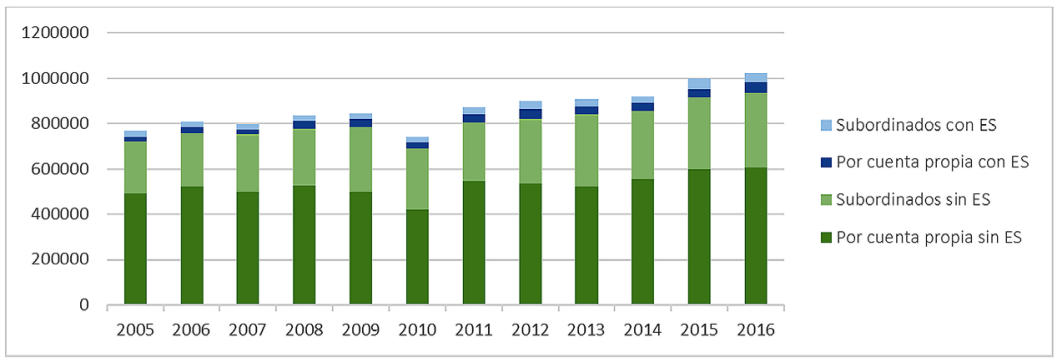

Fuente: elaboración propia a partir de los microdatos de la Encuesta Nacional de Ocupación y Empleo (Inegi, 2016).

minación laboral. Por lo anterior, el Estado interviene a través de acciones compensatorias que en alguna medida buscan mitigar las brechas de desigualdad, las cuales se discutirán en la siguiente sección.

\section{El papel de las políticas públicas en la atención de las necesidades en la tercera edad}

Las acciones gubernamentales para la atención de problemáticas y demandas de grupos socialmente vulnerables, como es el caso de los adultos mayores, se constituye en la identificación de las causas y efectos que tiene una situación o condición específica en una población en particular para así incluirlo en la agenda pública. Posteriormente, se definen e implementan las estrategias para dar soluciones. Como menciona Aguilar (2013), estas acciones denominadas políticas públicas recogen las demandas de los sectores civiles y políticos para ser estructuradas en objetivos y acciones legalmente válidas. Idealmente aprovechan la información y el conocimiento que existe en una sociedad para seleccionar las acciones y las formas de organización capaces de producir los resultados esperados. Implícitamente incluyen la relación costo-beneficio en términos de los recursos públicos necesarios para su exitosa operación.

Sin embargo, Elder y Cobb (2014) señalan que este grupo históricamente ha sido empleado para la determinación de problemas sociales que derivan en políticas públicas y programas de asistencia social, basados más en la edad que en la posibilidad de proteger sus derechos. Prueba de ello es que este sector no cuenta con representación como grupo organizado para la construcción de la agenda de política social en nuestro país. 
En este sentido, en el contexto mexicano pueden ser identificados casos de programas públicos para los adultos mayores, que si bien atienen sus demandas y problemáticas actuales, no forman parte de una estrategia de prevención que evite la falta de libertades asociadas a la vulnerabilidad de este grupo de la población. En la tabla 1 se muestran algunos de los programas públicos que son implementados para dar apoyo a las demandas del grupo de adultos mayores.

Los apoyos son mayormente asistencialistas, ya que en esencia buscan disminuir los efectos adversos que conlleva la edad sin resolver el problema de manera estructural. Pareciera que son reflejo de la incapacidad gubernamental para garantizar a los trabajadores al término de su trayectoria laboral el acceso a una pensión o jubilación digna. Es decir, la alta informalidad laboral en conjunto con la reducida base de contribuyentes no sólo afecta a las finanzas públicas a corto plazo, sino también impide que los mexicanos vean retribuidas sus contribuciones a largo plazo en términos de protección social.

\section{La calidad del empleo como herramienta para caracterizar a grupos socialmente vulnerables}

El concepto de calidad de vida se integra multidimensionalmente por factores sociales, económicos, psicológicos, contextuales, afectivos, emocionales, de salud, seguridad, acceso a servicios y respeto de los derechos humanos, entre otros. Para efectos de la presente investigación, el análisis se concentra en la calidad del empleo como un factor que contribuye a mejorar la calidad de vida de las personas. Cabe destacar que este concepto, lejos de ser estático, está condicionado por diversos elementos, incluidos las etapas de la vida.

En ese sentido, las personas de la tercera edad tienen características distintas al ocupado promedio. Fundamentalmente, la expectativa inicial consiste en el acceso a una pensión en sustitución de la actividad laboral para solventar las necesidades económicas y en el mejor de los casos concentrarse en los demás aspectos señalados anteriormente. Sin embargo, las altas tasas de informalidad presentes en nuestro país tienen una seria repercusión en este grupo etario ya que condiciona a una porción de éste a permanecer laboralmente activos durante toda la vida. Desafortunadamente, las condiciones en las que participan en el mercado laboral son mayormente precarias y directamente e indirectamente afectan en aspectos económicos, psicológicos y de salud trastocando la calidad de vida.

El estudio de la calidad del empleo mayormente se refiere a la integración y valoración de los diferentes elementos involucrados en el empleo para 


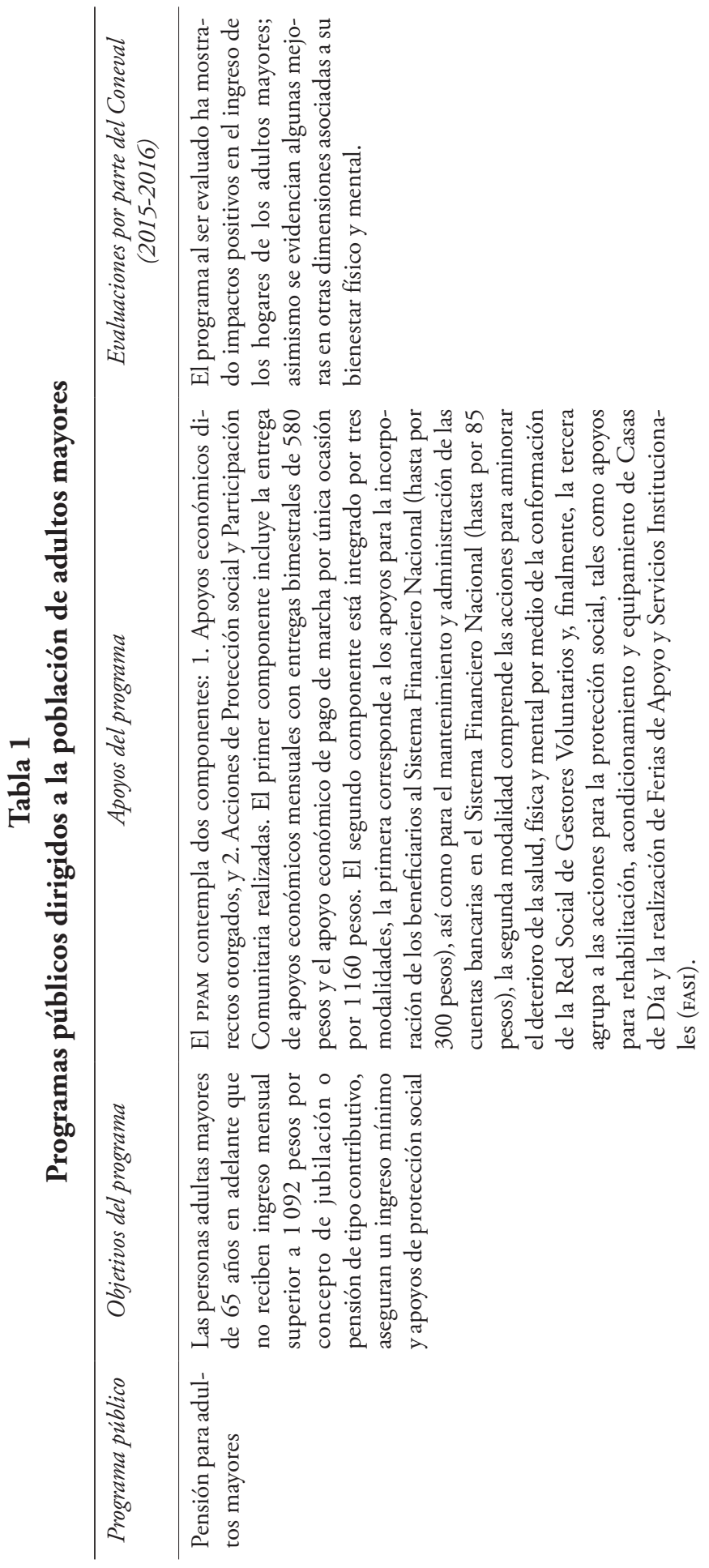




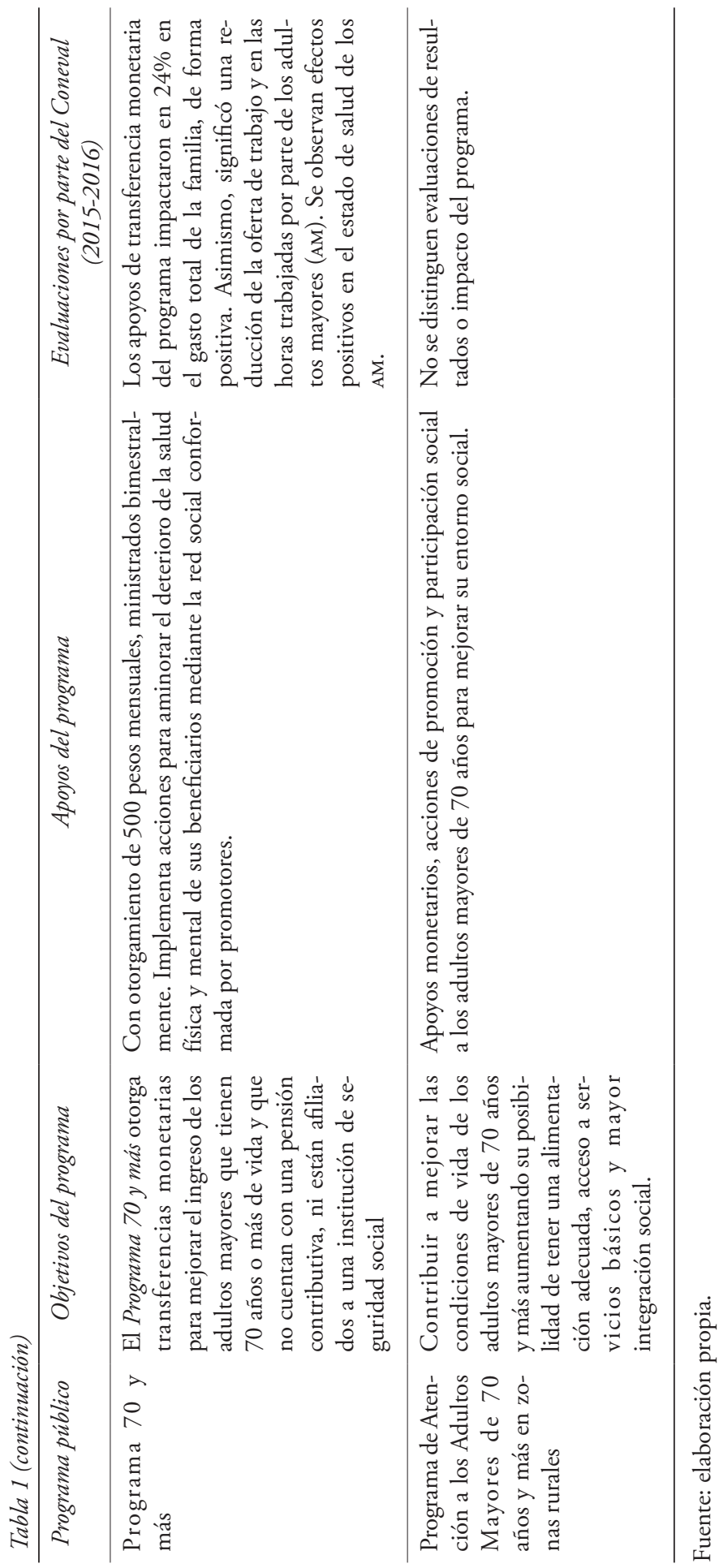


determinar el grado de bienestar que obtienen los trabajadores por su labor. Esto implica el análisis del propio mercado y el papel que desempeña la acción gubernamental a través de políticas públicas para incentivar el desarrollo (Ramos et al., 2015). Su conceptualización está fundamentada en la definición de empleo decente de la Organización Internacional del Trabajo. ${ }^{3}$ De manera que los estudios empíricos en su mayoría se enfocan en cuantificar las diferencias en la calidad del empleo en sectores productivos, grupos sociales, género y nivel educativo (Flores y Salas, 2015; Piras y Rucci, 2014; Gómez y Restrepo, 2013).

Sin embargo, se identificó un vacío en términos analíticos de las implicaciones del grupo de ocupados que estando dentro de la edad de jubilación todavía se encuentran trabajando, ya que estudios de este grupo etario se centran mayormente en el acceso a servicios de salud y aspectos asociados a la capacidad cognitiva y bienestar (Paredes, 2014; Gómez y Peña, 2013; Valdez et al., 2005).

Desde la perspectiva del desarrollo humano, Sen (2000) señala que el bienestar de las personas se encuentra en función de la ampliación de libertades a favor de la calidad de vida. Pineda (2007) acota este concepto a la calidad del empleo en términos de los beneficios obtenidos por el trabajo y su relación con las trayectorias laborales y vitales con respecto a la capacidad de toma de decisiones.

Sin embargo, el beneficio obtenido por el trabajo no es constante en el tiempo. Gómez et al. (2015) señalan la existencia de un ciclo de calidad del empleo, cuyo comportamiento es similar a una campana, dado que en la juventud la calidad del empleo es precaria y tiende a aumentar con la edad para posteriormente volver a disminuir. En ese sentido, las personas que llegan a la tercera edad sin la capacidad de acceder a una pensión o jubilación son más propensas al desempleo, lo cual da como resultado mayor precarización en caso de reincorporarse al mercado laboral ya que este grupo es más susceptible a la informalidad y al trabajo por cuenta propia (Acosta y Palacios, 2008).

Peláez y Ferrer (2001) señalan que las principales razones de tal precarización son problemas inherentes a la edad y su relación con la productividad, poca alfabetización digital, obsolescencia del conocimiento y alta propensión a la pensión. Complementariamente, Berstein y Tokman (2005) señalan que en este escenario la mujer es más vulnerable dado que sus trayectorias laborales son en promedio más intermitentes, debido a

\footnotetext{
${ }^{3}$ De acuerdo con la OIT (1999) "Significa también un trabajo suficiente, en el sentido de que todos deberían tener pleno acceso a las oportunidades de obtención de ingresos. Marca una pauta para el desarrollo económico y social con arreglo a la cual pueden cuajar la realidad del empleo, los ingresos y la protección social sin menoscabo de las normas sociales y de los derechos de los trabajadore”".
} 
roles tradicionales en la crianza que reducen la propensión de obtener una pensión.

La permanencia en el mercado de trabajo en la tercera edad no siempre es sinónimo de precariedad. La conjunción de factores tales como la viudez, buen estado de salud y contar con educación superior otorgan mayores beneficios que la pensión, por lo que el costo de oportunidad de dejar de trabajar es más alto (Gómez y Peña, 2013; Sala, 2012).

Por lo anterior, para valorar el grado de precarización del empleo en la tercera edad y su consecuente relación con la calidad de vida se calculó un índice multidimensional para profundizar en las implicaciones del trabajo posterior a la edad de jubilación.

\section{Metodología utilizada para cuantificar la calidad del empleo}

La construcción de índices multidimensionales supone la integración de variables que permiten explicar en alguna medida la complejidad inherente de la realidad social. Han sido varios los casos que han transitado de la medición unidimensional a la multidimensional para reflejar con mayor precisión la intrincada naturaleza de los problemas públicos.

Para ejemplificar, podemos destacar tres casos: la medición de la pobreza, la percepción sobre la seguridad pública y la calidad del empleo. Para el primer caso, la concepción tradicional se centraba en el ingreso familiar como factor clave para determinar la pobreza. Sin embargo, esta definición resultó insuficiente en términos de acción gubernamental para determinar el curso de acción adecuado para disminuir sus efectos. Por tal razón, en la actualidad se concibe como un problema multicausal que involucra aspectos como calidad de servicios públicos, acceso a salud y educación, alimentación, formalidad laboral y seguridad pública, entre otros.

Por su parte, la percepción sobre la seguridad pública se constituye por la integración de diferentes elementos implícitamente bajo un supuesto de información limitada e indeterminación. De tal manera que su construcción es resultado de experiencias previas, información presentada en los medios de comunicación, la retórica política, el marco legal, el grado de corrupción de las autoridades, entre otros factores.

Para el caso de la calidad del empleo, resulta insuficiente remitir su valoración exclusivamente al salario. Factores tales como la interdependencia de sectores económicos, la desregulación laboral y las nuevas formas de trabajo, derivadas de la globalización, requieren de una medición más precisa y compleja. En un sentido general, se han agregado como factores clave la estabilidad y la protección de los derechos laborales en un contexto 
basado en la flexibilidad de la mano de obra y el desarrollo de competencias laborales adaptables a las necesidades cambiantes del mercado.

Para efectos del presente escrito, se utilizó un índice de calidad del empleo basado en conjuntos de lógica difusa integrando las variables que se muestran a continuación:

\section{Indice de calidad del empleo}

$=f\left(\begin{array}{l}\text { Caracteristicas de la remuneración, estabilidad laboral, } \\ \text { prestaciones al corto plazo, prestaciones al largo plazo }\end{array}\right)$

Donde:

\section{Características de la remuneración}

$=f$ (Salario, número de empleos, duración de la jornada laboral)

Estabilidad laboral $=f$ (Tipo de contrato, capacidad de afiliación sindical)

Prestaciones de corto plazo $=f$ (Aguinaldo, vacaciones con goce de sueldo, reparto de utilidades, seguro de gastos médicos, préstamos personales/caja de ahorros)

Prestaciones de largo plazo $=f$ (Fondo para el retiro, seguro de vida, crédito para vivienda)

La construcción de dicho índice y sus respectivos subíndices se basa en conjuntos de lógica difusa, los cuales forman parte de la metodología de análisis cualitativo comparado ( $Q C A$ por sus siglas en inglés). Este tipo de sistemas permiten la integración no lineal de variables para así reflejar el comportamiento complejo de un fenómeno social. En este caso se utiliza para la construcción de un índice multidimensional, aunque no es su única aplicación.

En principio, la lógica difusa o borrosa, parte de dos premisas: el grado de pertenencia a un conjunto y la capacidad de pertenencia múltiple. En lo que respecta a la primera característica, el paradigma tradicional acota a clasificar los valores observados a un conjunto en particular de manera dicotómica (pertenece o no pertenece); por ejemplo, las personas de la tercera edad son aquellas con 65 años o más. Por su parte, el pensamiento difuso asigna grados de pertenencia al interior de cada conjunto para matizar en qué medida cada valor observado forma parte de la clasificación. Para ejemplificar, supongamos que empleamos como referencia la expectativa de vida en 2015 (74.9 años $\approx 75$ ). De manera que un sujeto con 75 años tendría una pertenencia total $(=1)$ al grupo de 
adultos mayores; mientras que una persona de 60 años tendría una pertenencia menor a este grupo (un valor mayor a 0 y menor a 1 ) puesto que es más "joven" con respecto del punto de referencia para un adulto mayor. De manera que ambos pertenecen al mismo grupo aunque no en la misma medida. Cabe destacar que el grado de pertenencia está relacionado con la función que utilicemos para describir el comportamiento del conjunto, es decir, puede ser lineal, logarítmico, exponencial e inclusive tipo escalón.

La segunda característica se refiere a la posibilidad de que un valor observado pertenezca simultáneamente a dos o más conjuntos. Esto resulta particularmente útil en los valores de intersección entre grupos, ya que la agregación del grado de pertenencia determina la valoración total dotando a la medición de mayor precisión. Para clarificar, la gráfica 4 muestra un conjunto difuso para representar la edad con respecto a dos grupos de edad: adultos y adultos mayores.

Como se ilustra, este conjunto está conformado por dos funciones: "adultos" y "adultos mayores". La mayor pertenencia a la segunda función se presenta en valores iguales o mayores a 75 ańos (punto A). Por su parte, el punto $B$ representa un valor (70 ańos) que pertenece al grupo de "adultos mayores" pero en menor grado que el punto A, ya que en este caso la pertenencia es igual a 0.5 . Por su parte, el punto $\mathrm{C}$ representa la pertenencia simultánea a los grupos "adultos" y "adultos mayores" (ambos en valores cercanos a 0.3) puesto que el valor de 67 ańos está cercano a la frontera entre grupos. Como se señaló anteriormente, esta diferenciación

\section{Gráfica 4}

Conjunto difuso para representar la pertenencia al grupo de adultos mayores

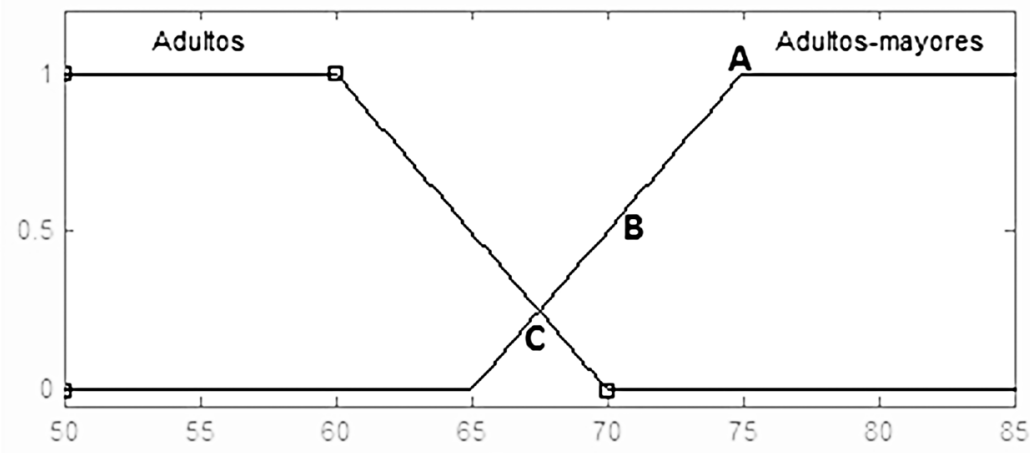

Fuente: elaboración propia. 
al interior de cada grupo permite mayor precisión asignando superioridad técnica a este tipo de herramientas.

En un segundo momento, el sistema de inferencia difuso requiere además de la definición de las variables y, su comportamiento, de reglas que permitan establecer los diferentes escenarios esperados a partir de la interacción entre variables. Para clarificar, supongamos dos variables asociadas a la calidad del empleo: salario (con funciones etiquetadas como "malo", "regular" y "bueno") y la jornada laboral (lingüísticamente en funciones "corta", "normal" y "larga"). De manera que las tablas de verdad describen a priori los diferentes escenarios y sus resultados. Es decir, de manera intuitiva la combinación de salario "bueno" y jornada "corta" supone mayor bienestar puesto que los sujetos adquieren mayores libertades tanto en capacidades económicas como en tiempo libre. Complementariamente, la condición más precaria se presenta con un salario "malo" y una jornada "larga". Asimismo, las demás combinaciones describirían los resultados intermedios.

En resumen, este tipo de herramientas posibilitan la creación de una "regla de medición", basada en la conjunción del comportamiento individual de cada variable y su interacción con las demás. Cabe señalar que su construcción parte del conocimiento sustantivo que se nutre de datos, tendencias, teorías, conceptos y testimonios de expertos. Como resultado se genera un dato continuo que está en función de los grados de pertenencia a las funciones y las tablas de verdad. En el anexo 1 se muestran a detalle las variables, funciones y tablas de verdad utilizadas para calcular el índice y los subíndices de calidad del empleo.

A fin de realizar el cálculo de los índices para valorar la calidad del empleo se utilizaron los microdatos de la Encuesta Nacional de Ocupación y Empleo (ENOE) durante el periodo 2005-2016. ${ }^{4}$ Se seleccionó esta base de datos considerando los siguientes aspectos: 1 . Porque permitió contar con la completitud de los aspectos planteados en los cuatro subejes de análisis (características de la remuneración, estabilidad laboral, prestaciones de corto plazo y prestaciones de largo plazo); 2. Por la disponibilidad de información en el tiempo, en este caso para establecer series de tiempo de 2005 a 2016 y; 3. Por su carácter oficial y su orientación específica al empleo y las condiciones laborales.

\footnotetext{
${ }^{4}$ Debido a que el cuestionario ampliado de la ENOE se aplica sólo en un trimestre cada año los datos utilizados corresponden al primer trimestre para 2005, 2009, 2010, 2011, 2012, 2013, 2014, 2015 y 2016; mientras que para 2006, 2007 y 2008 provienen del segundo trimestre.
} 


\section{Análisis}

El concepto de vulnerabilidad tiene repercusiones en los diferentes ámbitos de la vida de los sujetos. En el presente escrito, el foco de atención se centra en la calidad del empleo en personas de la tercera edad para dimensionar uno de los elementos que validan a este grupo social como vulnerable. En principio, el salario es identificado como variable central en la calidad del empleo. Se destaca que para efectos analíticos, los valores del salario están deflactados empleado como base el 2005 siendo aplicable también para el cálculo del subíndice de características de la remuneración.

Para el grupo de ocupados de 66-70 años se identifica que existe una tendencia a la precarización. Inclusive el segmento con mayor educación ha visto reducida su capacidad adquisitiva a lo largo de los últimos doce años. Asimismo, para el caso del grupo que no cuenta con educación superior la vulnerabilidad ha sido una constante debido a que el ingreso promedio es cercano al salario mínimo. A lo anterior hay que considerar que los ocupados con menor educación representan más de $90 \%$ de la masa laboral de este grupo etario como se ilustró en la gráfica 3. En la gráfica 5 se muestra la evolución de esta variable en el periodo 2005-2016.

En segunda instancia, la duración de jornada laboral permite dimensionar la relación salario-tiempo de dedicación. Para el caso del grupo analizado se presenta una clara tendencia a dedicar más tiempo al trabajo. Asimismo, durante el periodo estudiado la jornada siempre estuvo dentro de la clasificación de jornada normal de tiempo completo. Esto supone precariedad puesto que este grupo cuenta en términos generales

\section{Gráfica 5}

Salario de los ocupados de 66-70 ańos por tipo de empleo y educación. Salarios deflactados a valores de 2005 (2005=100)

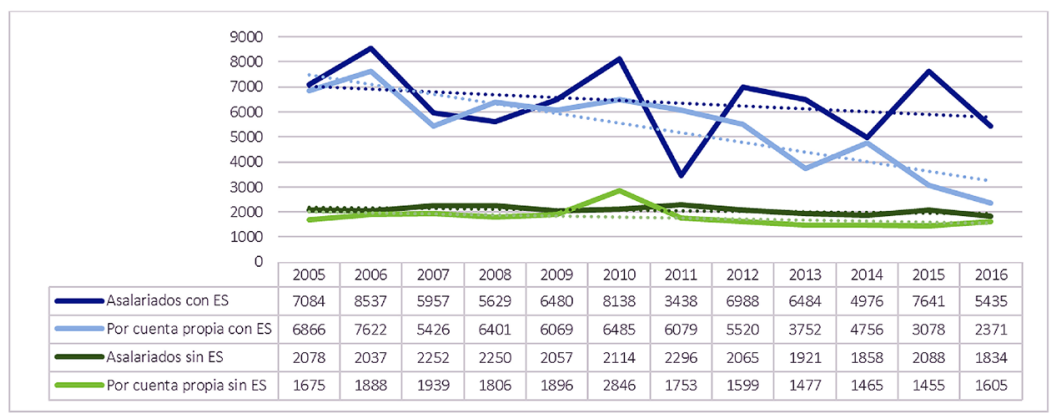

Fuente: elaboración propia a partir de los microdatos de la Encuesta Nacional de Ocupación y Empleo (Inegi, 2016). 
con capacidades físicas y mentales mermadas propias de la edad. En la gráfica 6 se muestra la evolución de esta variable en el tiempo.

\section{Gráfica 6 \\ Duración de la jornada laboral de los ocupados de 66 a 70 años}

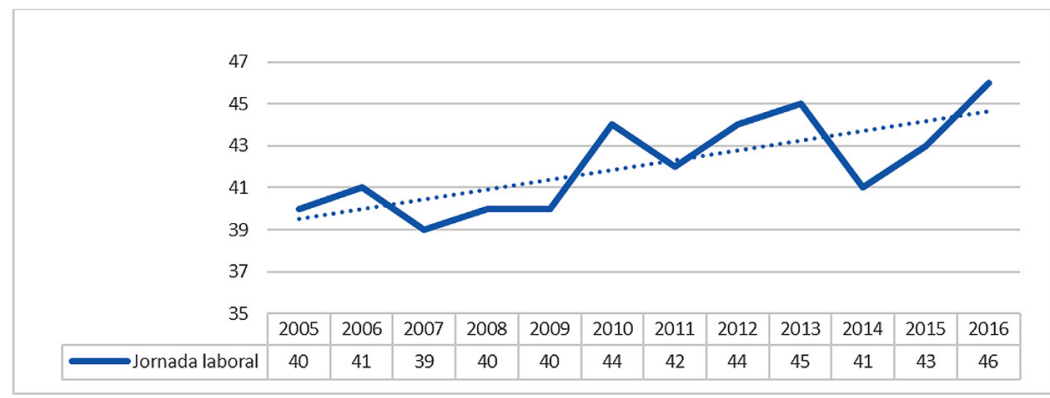

Fuente: elaboración propia a partir de los microdatos de la Encuesta Nacional de Ocupación y Empleo (Inegi, 2016).

El desgaste que supone una jornada laboral de tiempo completo tiene repercusiones en la salud de los trabajadores, por lo que es relevante destacar que aproximadamente $10 \%$ de los ocupados en esta cohorte acceden a servicios de salud por el empleo. Esto los sitúa en una clara condición de vulnerabilidad tomando en cuenta que las principales causas de muerte en personas mayores a 60 años están relacionadas con enfermedades crónicas. De acuerdo con la Secretaría de Salud, en 2016, las principales causas fueron las enfermedades del corazón (26.13\%) y la diabetes mellitus (16.39\%). Cabe destacar que paralelamente opera el programa Seguro Popular, el cual funciona como un programa compensatorio que proporciona acceso a servicios de salud no ligado al empleo. En la gráfica 7 se muestra la cobertura en el acceso a servicios de salud por el empleo para el periodo 2005-2016.

Complementariamente, la trayectoria laboral culmina con la jubilación de los trabajadores. Sin embargo, la generalizada informalidad laboral en nuestro país impide que existan condiciones óptimas para estructuralmente ceder el relevo laboral a las generaciones más jóvenes. Prueba de lo anterior, es que menos de 10\% de los ocupados en la cohorte de 66-70 años tiene acceso al fondo para el retiro tal como se ilustra en la gráfica 8.

Por lo anterior, se podría esperar que la mayoría de este grupo etario continuará trabajando en los próximos años. Como resultado es posible que se suscite mayor informalidad laboral y por tanto mayor precariedad misma que repercutirá directamente en detrimento de la calidad de vida 


\section{Gráfica 7 \\ Acceso a servicios de salud por el empleo de los ocupados de 66-70 ańos}

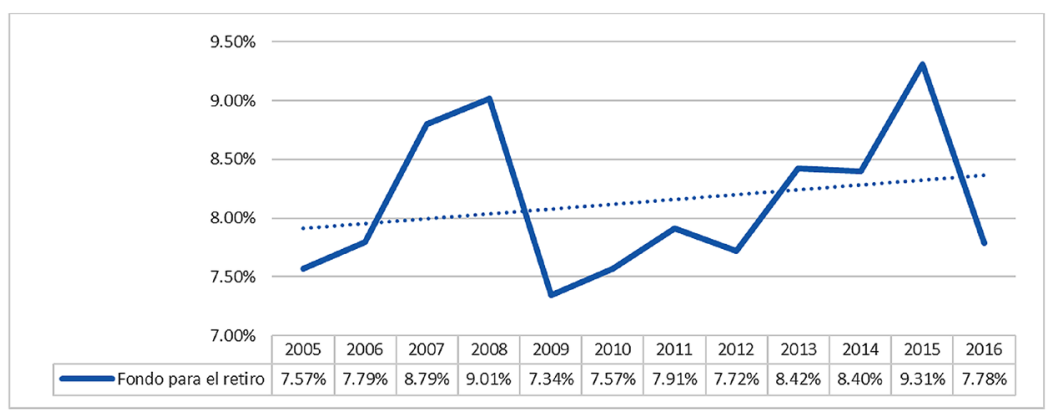

Fuente: elaboración propia a partir de los microdatos de la Encuesta Nacional de Ocupación y Empleo (Inegi, 2016).

\section{Gráfica 8}

\section{Acceso a fondo para el retiro de los ocupados de 66-70 años}

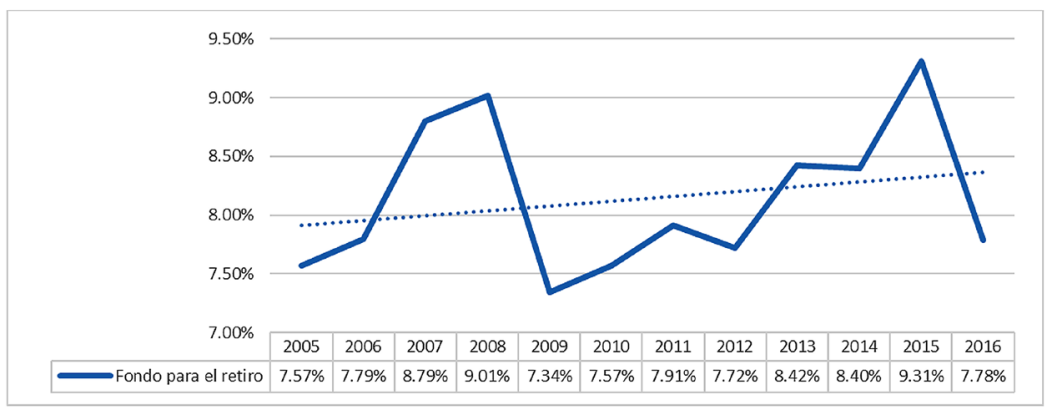

Fuente: elaboración propia a partir de los microdatos de la Encuesta Nacional de Ocupación y Empleo (Inegi, 2016).

dado que, como se señaló, el acceso a servicios de salud es limitado y los salarios son más bajos con respecto al promedio de los ocupados.

En un sentido más integral, al considerar la calidad del empleo resulta evidente el al to grado de vulnerabilidad que sufren los adultos mayores. Como ya se señaló, la mayoría de los ocupados no cuentan con estudios superiores, lo cual restringe el tipo de puestos a los que este tipo de trabajadores puede acceder. Como se ilustra en la gráfica 9, sólo el reducido grupo de asalariados con educación superior cuenta con condiciones para afrontar con dignidad la denominada tercera edad.

Cabe destacar que en términos estructurales la calidad del empleo del grupo analizado no mejoró a lo largo de doce ańos proporcionando evi- 
Gráfica 9

\section{Calidad del empleo de los ocupados de 66-70 años por tipo de empleo y educación}

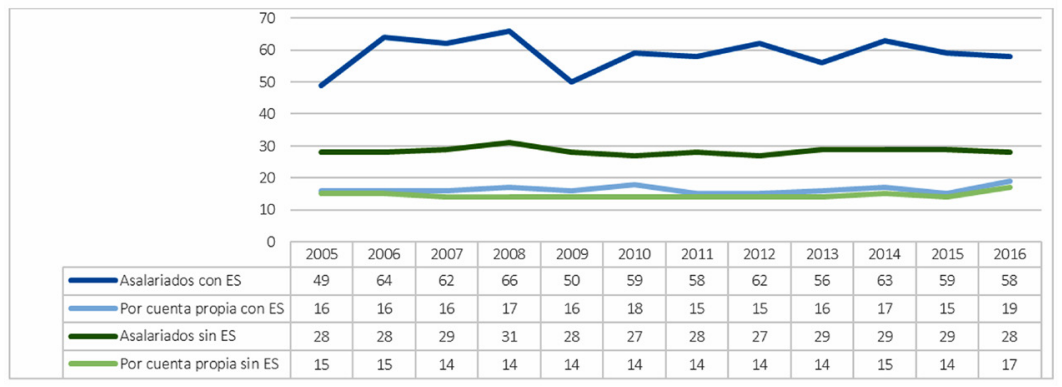

Fuente: elaboración propia a partir de los microdatos de la Encuesta Nacional de Ocupación y Empleo (Inegi, 2016).

dencia de que las políticas públicas y programas no han compensado la inequidad en este grupo. Tal desigualdad se vuelve más evidente cuando separamos la calidad del empleo en sus diferentes componentes. En la gráfica 10 se muestran las diferencias entre cuatro grupos de ocupados.

Como se observa, el grupo mayoritario depende primordialmente de la relación existente entre el salario-jornada laboral-número de empleos, es decir, la calidad del empleo de los adultos mayores se reduce al salario sin que exista otro elemento adicional que compense la alta precariedad laboral. Esto sitúa a este grupo en una alta vulnerabilidad puesto que no se identifica en términos temporales una mejora en sus condiciones.

Lo anterior posibilita la identificación de cuatro tipos de ocupados a partir de la combinación del tipo de empleo y el grado de escolaridad. El grupo más representativo es el de trabajadores por cuenta propia sin educación superior, que en 2017 representa 59.38\% de los ocupados de 66 a 70 años. En segunda instancia están los ocupados con educación superior con $32.08 \%$, mientras que los trabajadores asalariados sin educación superior con $4.49 \%$ de representación. En conjunto estos tres grupos comparten una alta precariedad laboral ya que además de dedicar jornadas laborales mayores a las 40 horas a la semana el beneficio que obtienen radica casi de manera exclusiva en salarios bajos -cercanos al mínimo- y sin prestaciones, acceso a servicios de salud o fondo para el retiro. Desafortunadamente estos grupos conforman $95.95 \%$ del total. Como se señaló se parte de la idea de que la precariedad laboral impacta negativamente en la calidad de vida de las personas contribuyendo a que se encuentren en condiciones de vulnerabilidad.

El único grupo alejado de esta situación son los asalariados con educación superior cuyas condiciones laborales están inclusive por encima de 


\section{Gráfica 10}

\section{Composición de la calidad del empleo de los ocupados de 66 a 70 años en 2016}

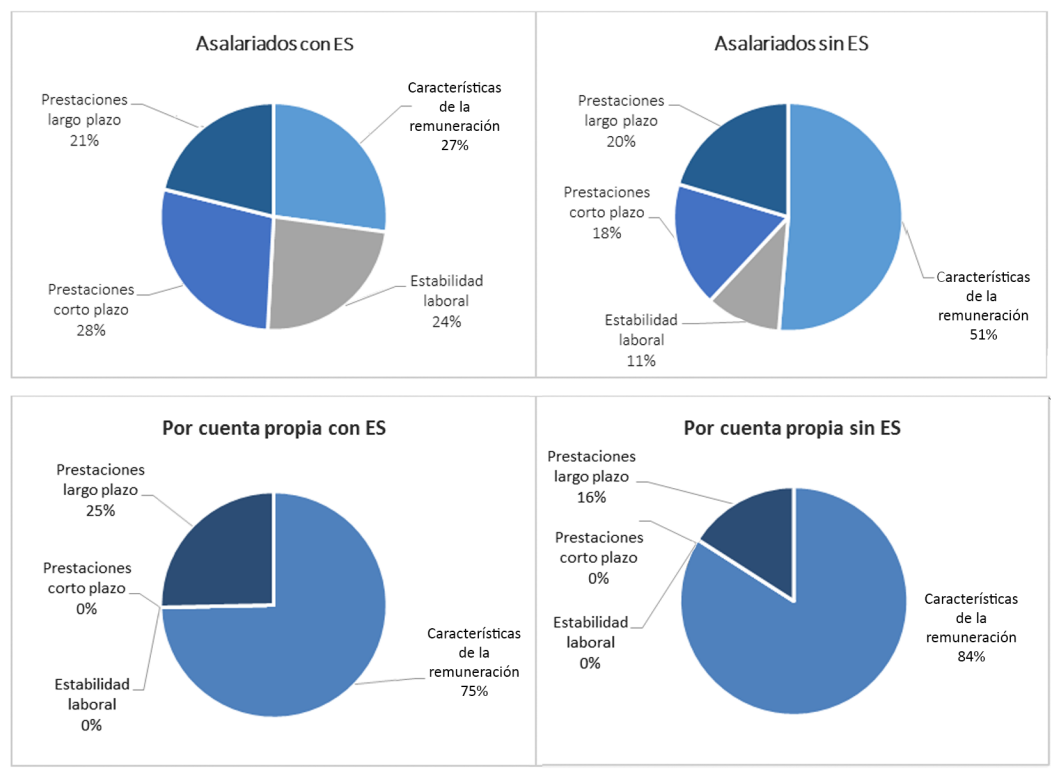

Fuente: elaboración propia a partir de los microdatos de la Encuesta Nacional de Ocupación y Empleo (Inegi, 2016).

la media de la masa laboral con salarios promedio de 7684 pesos en 2016. Se podría asumir que este grupo encuentra en el empleo una fuente de bienestar que impacta positivamente en su calidad de vida, al menos en lo que respecta a un cierto grado de autonomía económica.

\section{Conclusiones}

A partir de la evidencia presentada se reafirma la noción de que los adultos mayores ocupados han visto mermadas sus condiciones laborales, en este caso, a lo largo de los doce años empleados para el análisis. Es importante destacar que la precariedad laboral tiene su origen en la generalizada informalidad laboral en nuestro país. Esto tiene como consecuencia la incapacidad de los adultos mayores para finalizar la trayectoria laboral con una pensión o jubilación. Más aún, los ocupados en esta cohorte mayormente no cuentan con fondo para el retiro, lo cual combinado con salarios bajos y jornadas laborales cercanas e incluso mayores 
a 40 horas a la semana contundentemente los sitúa en condición de vulnerabilidad.

Bajo esa mirada, la capacidad de integración multidimensional del índice empleado posibilita contar con un panorama con mayores matices, entre los cuales podemos destacar tres aspectos. El primero de ellos se refiere a que inclusive en este grupo etario la educación superior continúa siendo una variable significativa para explicar los empleos con mejor calidad. Asimismo, esto contrasta con la muy baja cobertura de este nivel educativo para esta generación con respecto de la actual cobertura, cercana a 30\%, en la cohorte de 20 a 24 ańos. En segundo lugar, permite comprobar que las condiciones laborales de este grupo de ocupados mayormente se concentran en el salario, ya que se observó que en términos de estabilidad y prestaciones sólo los asalariados cuentan con algunos de los elementos empleados para el cálculo del índice. Sin embargo, el grupo mayoritario es aquel cuyo trabajo es por cuenta propia cuya denominación pareciera más cercana a la informalidad. En tercer lugar, se observa que la calidad del empleo permanece prácticamente constante a lo largo del periodo estudiado. De manera que si dicho valor es asociable a empleos precarios la propia inercia apuntaría a pensar que los ocupados en esta condición están potencialmente obligados a continuar trabajando durante toda su vida.

En este sentido, se evidencia que la calidad de vida de este tipo de grupos sociales se ve mermada desde la materialización de la vulnerabilidad reflejada en precariedad laboral y vulnerabilidad social.

Asimismo, se puede comprobar que para estas generaciones la política social no generó beneficio suficiente en términos estructurales puesto que como se señaló en la introducción este grupo ha vivido en condiciones generalizadas de pobreza durante toda su vida. Prueba de lo anterior, son los matices de la política social cuya orientación es clara hacia el asistencialismo. Para ejemplificar, programas como el Seguro Popular ofrecen acceso a salud no ligado al empleo considerando que las dos principales causas de muerte del grupo de mayores de 60 años provienen de enfermedades crónicas: enfermedades del corazón y diabetes mellitus.

El programa 65 y más otorga apoyo económico a cierto grupo de los adultos mayores. Sin embargo, en términos generales no se identifican esfuerzos gubernamentales significativos para revertir la alta tasa de informalidad que deriva en la tercera edad en precariedad laboral. Igualmente es evidente que en términos de política pública no se cumple con el ciclo ya que los procesos de evaluación son indispensables para la mejora continua de los programas. Esta carencia impacta negativamente en el caso concreto de los adultos mayores. 
En un sentido más amplio, se identificó que el problema público en cuestión no se presenta al momento en que las personas llegan a la tercera edad sino que es la culminación de otros problemas de orden estructural. Es decir, la combinación de desigualdad en el acceso a las oportunidades educativas, bajos salarios y altas tasas de informalidad presentes desde la juventud se arrastra hasta la tercera edad, lo cual agudiza sus consecuencias y sitúa a este grupo como naturalmente vulnerable. Por tal razón se asume en el presente escrito que la precariedad laboral es un factor clave para entender las condiciones de calidad de vida de este grupo social.

Por otra parte, es relevante mencionar que la metodología empleada para el análisis cobra pertinencia bajo la idea de que con este tipo de metodologías robustas se pueden identificar elementos difusos e inconsistentes para darles explicaciones nítidas. Los conjuntos difusos de forma particular en este trabajo permitieron hacer un análisis con mayor precisión, esto en virtud de que en la actualidad el estudio de los fenómenos sociales y, específicamente, del análisis de grupos socialmente vulnerables se requiere del manejo de la multidimensionalidad. 


\section{Anexos}

Anexo 1. Conjuntos difusos empleados para la valoración de la calidad del empleo.

a) Subíndice características de la remuneración
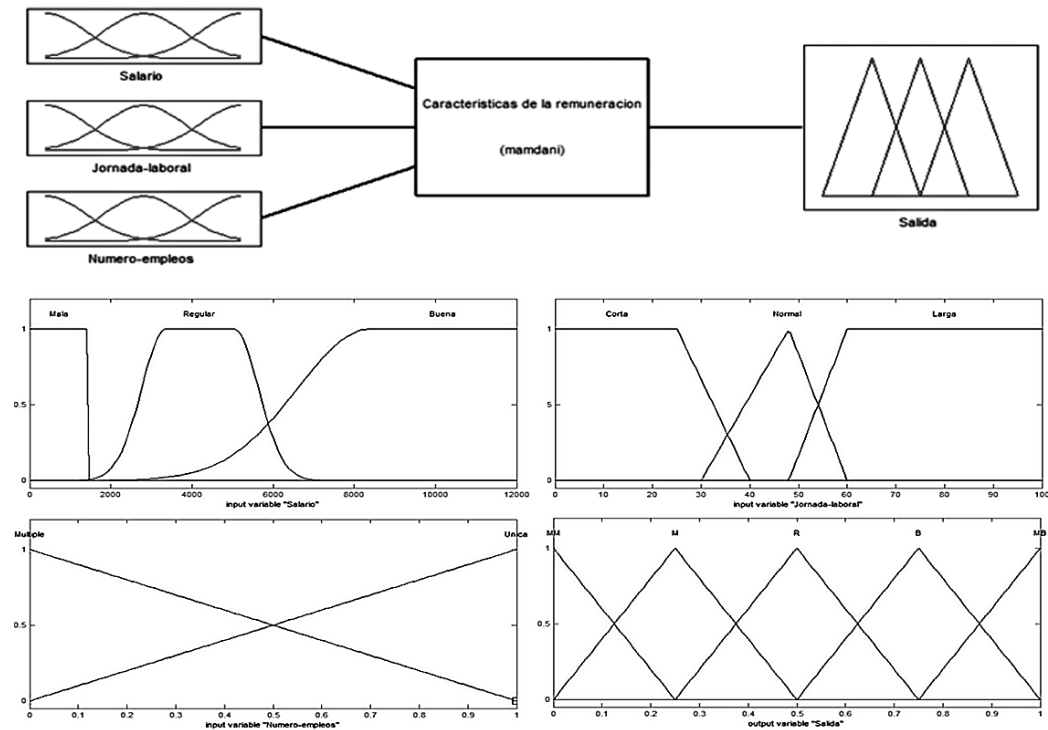

1. If (Salario is Buena) and (Jornada-laboral is Corta) and (Numero-empleos is Unica) then (Salida is MB) (1) 2. If (Salario is Buena) and (Jornada-laboral is Normal) and (Numero-empleos is Unica) then (Salida is B) (1) 3. If (Salario is Buena) and (Jornada-laboral is Larga) and (Numero-empleos is Unica) then (Salida is B) (1)

4. If (Salario is Buena) and (Jornada-laboral is Corta) and (Numero-empleos is Multiple) then (Salida is B) (1)

5. If (Salario is Buena) and (Jornada-laboral is Normal) and (Numero-empleos is Multiple) then (Salida is R) (1)

6. If (Salario is Buena) and (Jornada-laboral is Larga) and (Numero-empleos is Multiple) then (Salida is M) (1)

7. If (Salario is Regular) and (Jornada-laboral is Corta) and (Numero-empleos is Unica) then (Salida is B) (1)

8. If (Salario is Regular) and (Jornada-laboral is Normal) and (Numero-empleos is Unica) then (Salida is R) (1)

9. If (Salario is Regular) and (Jornada-laboral is Larga) and (Numero-empleos is Unica) then (Salida is R) (1)

10. If (Salario is Regular) and (Jornada-laboral is Corta) and (Numero-empleos is Multiple) then (Salida is R) (1)

11. If (Salario is Regular) and (Jornada-laboral is Normal) and (Numero-empleos is Multiple) then (Salida is $M$ ) (1)

12. If (Salario is Regular) and (Jornada-laboral is Larga) and (Numero-empleos is Multiple) then (Salida is MM) (1)

13. If (Salario is Mala) and (Jornada-laboral is Corta) and (Numero-empleos is Unica) then (Salida is B) (1)

14. If (Salario is Mala) and (Jornada-laboral is Normal) and (Numero-empleos is Unica) then (Salida is R) (1)

15. If (Salario is Mala) and (Jornada-laboral is Larga) and (Numero-empleos is Unica) then (Salida is M) (1)

16. If (Salario is Mala) and (Jornada-laboral is Corta) and (Numero-empleos is Multiple) then (Salida is M) (1)

17. If (Salario is Mala) and (Jornada-laboral is Normal) and (Numero-empleos is Multiple) then (Salida is MM) (1)

18. If (Salario is Mala) and (Jornada-laboral is Larga) and (Numero-empleos is Multiple) then (Salida is MM) (1)

Fuente: elaboración de los autores. 
b) Subíndice estabilidad laboral
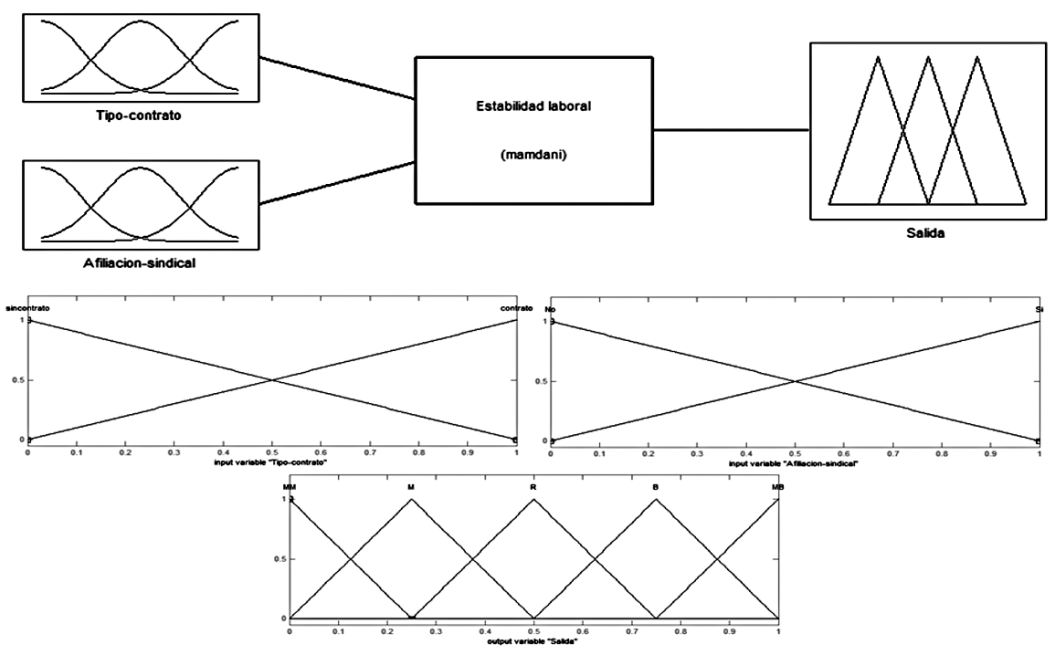

1. If (Tipo-contrato is sincontrato) and (Afiliacion-sindical is No) then (Salida is MMM) (1)

2. If (Tipo-contrato is contrato) and (A filiacion-sindical is Si) then (Salida is MB) (1)

3. If (Tipo-contrato is contrato) and (Afiliacion-sindical is No) then (Salida is R) (1)

4. If (Tipo-contrato is sincontrato) and (Afiliacion-sindical is Si) then (Salida is R) (1)

Fuente: elaboración de los autores. 
c) Subíndice prestaciones corto plazo
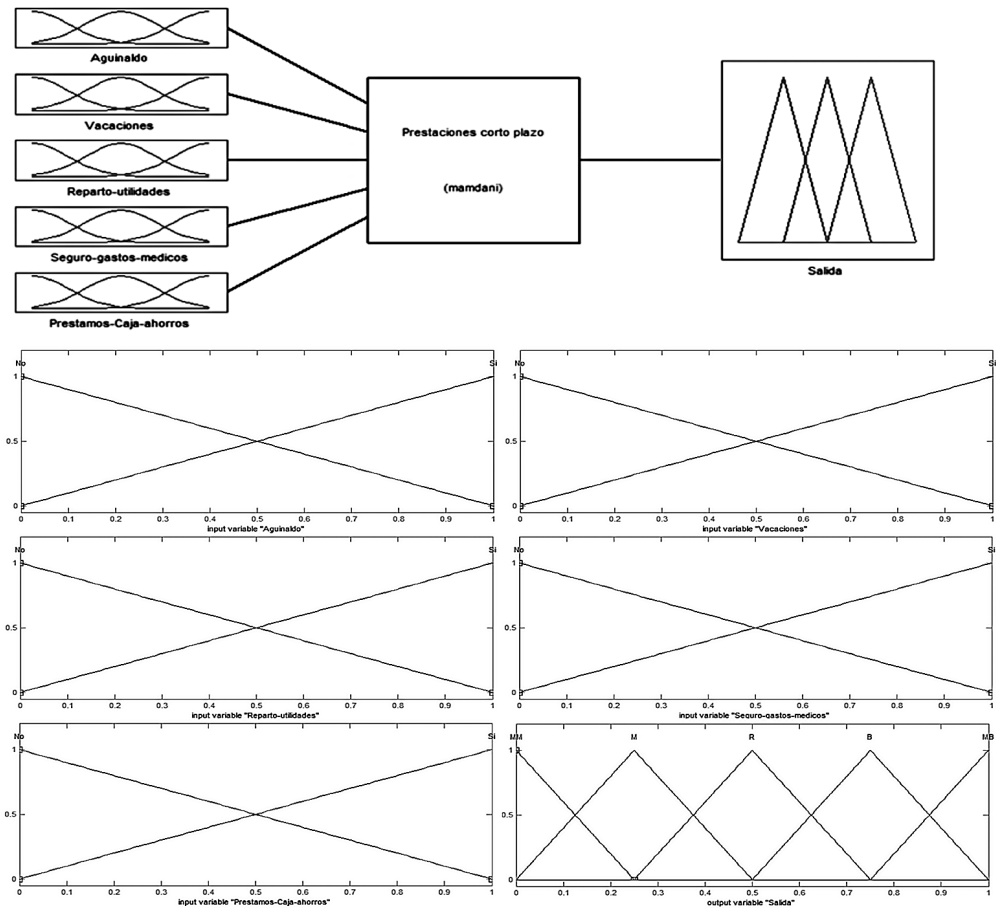

1. If (Aguinaldo is $\mathrm{Si}$ ) and (Vacaciones is $\mathrm{Si}$ ) and (Reparto-utilidades is $\mathrm{Si}$ ) and (Seguro-gastos-medicos is $\mathrm{Si}$ ) and (Prestamos-Caja-ahorros is $\mathrm{Si}$ ) then (Salida is MB) (1) 2. If (Aguinaldo is $\mathrm{Si}$ ) and (Vacaciones is $\mathrm{Si}$ ) and (Reparto-utitidades is $\mathrm{Si}$ ) and (Seguro-gastos-medicos is $\mathrm{Si}$ ) and (Prestamos-Caja-ahorros is No) then (Salida is $M$ MB) (1) 3. If (Aguinaloo is $\mathrm{SI}$ ) and (Vacaciones is $\mathrm{Si}$ ) and (Reparto-utiddades is $\mathrm{Si}$ ) and (Seguro-gastos-medicos is $\mathrm{No}$ ) and (Prestamos-Caja-ahorros is $\mathrm{SI}$ ) then (Salida is $M B$ ) ( $(1)$

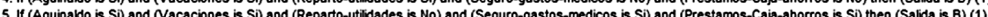

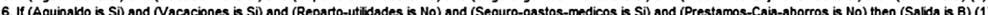
7 . If (Aguinato is Si) and (

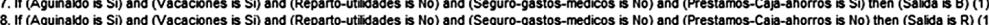
9. If (Aguinaldo is $\mathrm{Si}$ ) and (Vacaciones is No) and (Reparto-utibdades is $\mathrm{Si}$ ) and (Seguro-gastos-medicos is Si) and (Prestamos-Caja-ahorros is Si) then (Salida is B) (1) 10. If (Aguinaldo is $\mathrm{Si}$ ) and (Vacaciones is No) and (Reparto-utilidades is Si) and (Seguro-gastos-medicos is Si) and (Prestamos-Caja-ahorros is No) then (Salida is $\mathrm{B}$ ) (1) 11. If (Aguinaldo is Si) and (Vacaciones is No) and (Reparto-utilidades is Si) and (Seguro-gastos-medicos is No) and (Prestamos-Caja-ahorros is $S_{i}$ ) then (Salida is B) (1) 12. If (Aguinaldo is $\mathrm{Si}$ ) and (Vacaciones is No) and (Reparto-utilidades is $\mathrm{Si}$ ) and (Seguro-gastos-medicos is No) and (Prestamos-Caja-ahorros is No) then (Salida is R) (1) 13. If (Aguinaldo is $\mathrm{Si}$ ) and (Vacaciones is No) and (Reparto-utilidades is No) and (Seguro-gastos-medicos is $\mathrm{Si}$ ) and (Prestamos-Caja-ahorros is $\mathrm{Si}$ ) then (Salida is $\mathrm{R}$ ) (1) 14. If (Aguinaldo is $\mathrm{Si}$ ) and (Vacaciones is No) and (Reparto-utilidades is No) and (Seguro-gastos-medicos is $\mathrm{Si}$ ) and (Prestamos-Caja-ahorros is No) then (Salida is $\mathrm{R}$ ) (1) 15. If (Aguinaldo is Si) and (Vacaciones is No) and (Reparto-utilidades is No) and (Seguro-gastos-medicos is No) and (Prestamos-Caja-ahorros is Si) then (Salida is R) (1) 16. If (Aguinaldo is $\mathrm{Si}$ ) and (Vacaciones is No) and (Reparto-utilidades is $\mathrm{No}$ ) and (Seguro-gastos-medicos is $\mathrm{No}$ ) and (Prestamos-Caja-ahorros is No) then (Salida is $\mathrm{M}$ ) (1) 17. If (Aguinaldo is No) and (Vacaciones is $\mathrm{Si}$ ) and (Reparto-utilidades is $\mathrm{Si}$ ) and (Seguro-gastos-medicos is $\mathrm{SI}$ ) and (Prestamos-Caja-ahorros is Si) then (Salida is B) (1) 18. If (Aguinaldo is No) and (Vacaciones is $\mathrm{Si}$ ) and (Reparto-utilidades is $\mathrm{Si}$ ) and (Seguro-gastos-medicos is $\mathrm{SI}$ ) and (Prestamos-Caja-ahorros is No) then (Salida is $\mathrm{R}$ ) (1) 19. If (Aguinaldo is No) and (Vacaciones is $\mathrm{Si}$ ) and (Reparto-utilidades is $\mathrm{Si}$ ) and (Seguro-gastos-medicos is No) and (Prestamos-Caja-ahorros is $\mathrm{Si}$ ) then (Salida is $\mathrm{R}$ ) (1) 20. If (Aguinaldo is No) and (Vacaciones is $\mathrm{Si}$ ) and (Reparto-utilidades is $\mathrm{Si}$ ) and (Seguro-gastos-medicos is No) and (Prestamos-Caja-ahorros is No) then (Salida is R) (1) 21. If (Aguinaldo is No) and (Vacaciones is $\mathrm{Si}$ ) and (Reparto-utilidades is $\mathrm{No}$ ) and (Seguro-gastos-medicos is $\mathrm{Si}$ ) and (Prestamos-Caja-ahorros is $\mathrm{Si}$ ) then (Salida is R) (1) 22. If (Aguinaldo is No) and (Vacaciones is $\mathrm{Si}$ ) and (Reparto-utilidades is No) and (Seguro-gastos-medicos is $\mathrm{Si}$ ) and (Prestamos-Caja-ahorros is No) then (Salida is $M$ ) (1) 23. If (Aguinaldo is No) and (Vacaciones is $\mathrm{Si}$ ) and (Reparto-utilidades is No) and (Seguro-gastos-medicos is No) and (Prestamos-Caja-ahorros is Si) then (Salida is M) (1) 24. If (Aquinaldo is No) and (Vacaciones is Si) and (Reparto-utilidades is No) and (Sequro-aastos-medicos is No) and (Prestamos-Caia-ahorros is No) then (Salida is M) (1) 25. If (Aguinaldo is No) and (Vacaciones is No) and (Reparto-utilidades is Si) and (Seguro-gastos-medicos is Si) and (Prestamos-Caja-ahorros is Si) then (Salida is R) (1) 26. If (Aguinaldo is No) and (Vacaciones is No) and (Reparto-utilicades is Si) and (Seguro-gastos-medicos is $\mathrm{SI}$ ) and (Prestamos-Caja-ahorros is No) then (Salida is $\mathrm{M}$ ) (1) 27. If (Aguinaldo is No) and (Vacaciones is No) and (Reparto-vtidoades is SI) and (Seguro-gastos-medicos is No) and (Prestamos-Caja-ahorros is SI) then (Salida is M) (1) 28. If (Aguinaldo is No) and (Vacaciones is No) and (Reparto-utilidades is SI) and (Seguro-gastos-medicos is No) and (Prestamos-Caja-ahorros is No) then (Saida is $\mathrm{M}$ ) (1) 30. 31. 32. If (Aguinaldo is No) and (Nacaciones is No) and (Reparto-utilidades is No) and (Seguro-gastos-medicos is No) and (Prestamos-Caja-ahorros is No) then (Salida is MM) (1)

Fuente: elaboración de los autores. 
d) Subíndice prestaciones largo plazo
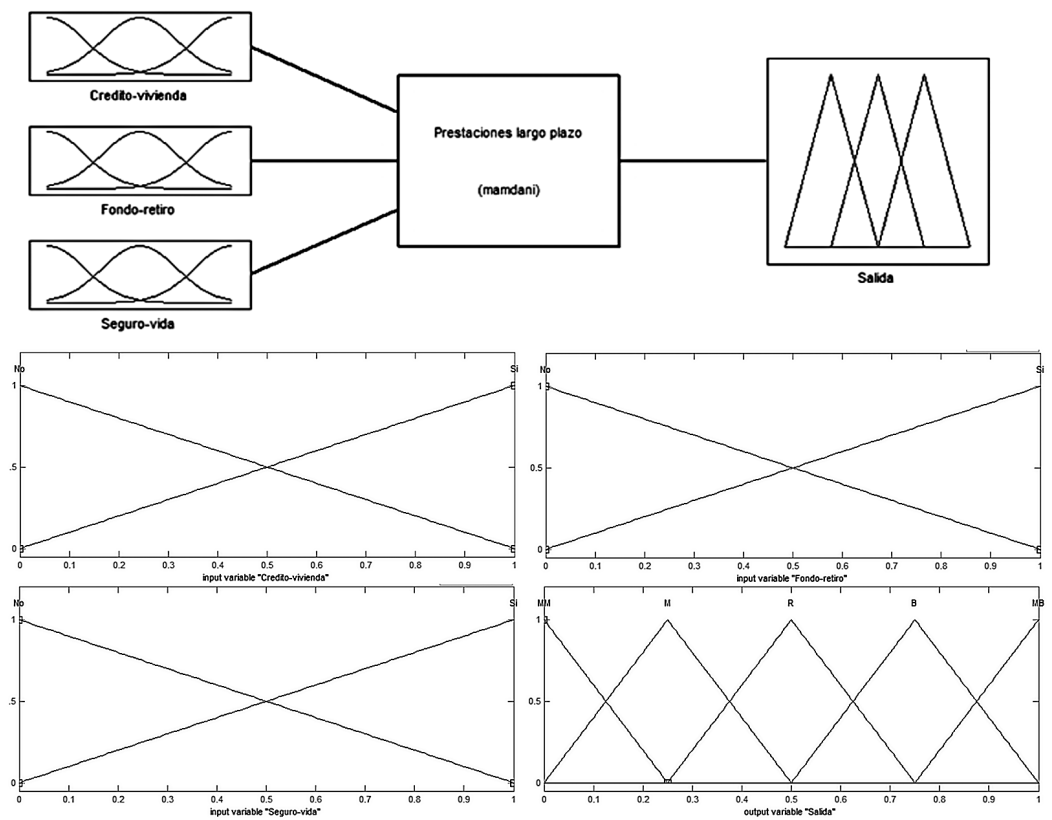

1. If (Credito-vivienda is Si) and (Fondo-retiro is Si) and (Seguro-vida is Si) then (Salida is MB) (1)

2. If (Credito-vivienda is $\mathrm{No}$ ) and (Fondo-retiro is No) and (Seguro-vida is No) then (Salida is MMI) (1)

3. If (Credito-vivienda is $\mathrm{No}$ ) and (Fondo-retiro is $\mathrm{No}$ ) and (Seguro-vida is Si) then (Salida is MMM) (1)

4. If (Credito-vivienda is No) and (Fondo-retiro is Si) and (Seguro-vida is No) then (Salida is R) (1)

5. If (Credito-vivienda is No) and (Fondo-retiro is Si) and (Seguro-vida is Si) then (Salida is B) (1)

6. If (Credito-vivienda is $\mathrm{Si}$ ) and (Fondo-retiro is $\mathrm{No}$ ) and (Seguro-vida is $\mathrm{No}$ ) then (Salida is R) (1)

7. If (Credito-vivienda is $\mathrm{Si}$ ) and (Fondo-retiro is $\mathrm{No}$ ) and (Seguro-vida is $\mathrm{Si}$ ) then (Salida is $\mathrm{B}$ ) (1)

8. If (Credito-vivienda is $\mathrm{Si}$ ) and (Fondo-retiro is $\mathrm{Si}$ ) and (Seguro-vida is No) then (Salida is B) (1)

Fuente: elaboración de los autores. 


\section{Fuentes consultadas}

Acosta, Clara y Nelly Palacios (2008), "Situación económica y bienestar psicológico en adultos mayores de Balancán, Tabasco", Horizonte Sanitario, 7 (3), Universidad Juárez Autónoma de Tabasco, Villahermosa, México, pp. 9-19.

Aguilar, Luis Fernando (2013), "Estudio introductorio", en Luis Fernando Aguilar (ed.), El estudio de las politicas públicas, Miguel Ángel Porrúa, Ciudad de México, México, pp. 15-74.

Berstein, Solange y Andrea Tokman (2005), "Brechas de ingreso entre géneros: ¿perpetuadas o exacerbadas en la vejez?", documento de trabajo número 8, Superintendencia de Administradoras de Fondos de Pensiones-Gobierno de Chile, Santiago, Chile.

Busso, Gustavo (2002), "Vulnerabilidad sociodemográfica en Nicaragua: un desafío para el crecimiento económico y la reducción de la pobreza”, Comisión Económica para América Latina y el Caribe, Santiago, Chile.

Elder, Charles y Roger Cobb (2014), "Formación de la agenda. El caso de la política de los ancianos", en Luis Fernando Aguilar (ed.), Problemas públicos y agenda de gobierno, Miguel Ángel Porrúa, Ciudad de México, México, pp. 77-104.

Flores, Lucio e Iván Alejandro Salas (2015), "Las brechas de género en la calidad del empleo en México. Una valoración basada en modelos de lógica difusa", Análisis Económico, 30 (75), Universidad Autónoma Metropolitana, Ciudad de México, México, pp. 89112.

Golovanevsky, Laura (2007), "Vulnerabilidad Social: Una propuesta para su medición en Argentina", Revista de Economía y Estadística, 45 (2), Instituto de Economía y Finanzas de la Universidad Nacional de Córdoba, Córdoba, Argentina, pp. 53-94.

Gómez, Mónica Sofía, Luis Armando Galvis-Aponte y Vicente Royuela (2015), "Calidad de vida laboral en Colombia: un índice multidimensional difuso", documento de trabajo número 230, Banco de la República-Banco Central de Colombia, Cartagena, Colombia. 
Gómez, Marcela Georgina y Julio Peña (2013), “Adultos Mayores. Un estudio socioeducativo en Ciudad Juárez, Chihuahua, México", Revista Interamericana de Educación de Adultos, 35 (2), Centro de Cooperación Regional para la Educación de Adultos en América Latina y el Caribe, Pátzcuaro, México, pp. 64-90.

Gómez, María Alejandra y David Restrepo (2013), "El trabajo precario, una realidad distante del trabajo decente", Katharsis, núm. 15, Institución Universitaria de Envigado, Envigado, Colombia, pp. 173-194.

Inegi (Instituto Nacional de Estadística y Geografía) (2016), "Encuesta Nacional de Ocupación y Empleo. Microdatos 2005-2016”, Inegi, Aguascalientes, México. <http://www.beta.inegi.org.mx/ proyectos/enchogares/regulares/enoe/>, 9 de diciembre de 2016.

Inegi (Instituto Nacional de Estadística y Geografía) (2015), "Encuesta Intercensal del Inegi en 2015”, Inegi, Aguascalientes, México, $<$ http://www.beta.inegi.org.mx/proyectos/enchogares/especiales/ intercensal/default.html>, 9 de diciembre de 2016.

Navarro Ornelas, Ana María y Raúl Sergio González-Ramírez (2010), "Los hogares con jefatura femenina y la calidad de vida. Chihuahua y Tijuana, 2005”, Estudios Demográficos y Urbanos, 25 (1), El Colegio de México, A. C., Ciudad de México, México, pp. 45-72.

OIT (Organización Internacional del Trabajo) (1999), Memoria del Director General: trabajo decente, Organización Internacional del Trabajo, Ginebra, Suiza.

Paredes, Mariana (2014), "Las personas mayores en el Uruguay: el perfil sociodemográfico y los desafíos para las políticas públicas", Notas de Población, núm. 98, Centro Latinoamericano y Caribeño de Demografía-División de Población de la Comisión Económica para América Latina y el Caribe, Santiago, Chile, pp. 41-74.

Peláez, Martha y Marcela Ferrer (2001), "Salud pública y los derechos humanos de los adultos mayores", Acta Bioethica, 7 (1), Centro Interdisciplinario de Estudios en Bioética de la Universidad de Chile, Santiago, Chile, pp. 143-155. 
Pineda, Javier (2007), "Calidad del empleo e inequidades de género", Revista CS Estudios sobre Latinoamérica y el Caribe con perspectiva global, núm.1, Universidad ICESI, Cali, Colombia, pp. 60-98.

Piras, Claudia y Graciana Rucci (2014), Chile: participación laboral femenina y calidad del empleo, Banco Interamericano de Desarrollo, Santiago, Chile.

Pizarro, Roberto (2001), La vulnerabilidad social y sus desafios: una mirada desde América Latina, Naciones Unidas-Comisión Económica para América Latina y el Caribe, Santiago, Chile.

Ramos, Joseph, Kirsten Sehnbruch y Jürgen Weller (2015), "Calidad del empleo en América Latina. Teoría y datos empíricos", Revista Internacional del Trabajo, 134 (2), Organización Internacional del Trabajo, Ginebra, Suiza, pp. 187-212.

Sala, Gabriela (2012), "Cobertura previsional, empleo y desempleo entre los adultos mayores argentinos", Revista Latinoamericana de Población, 6 (11), Asociación Latinoamericana de Población, Buenos Aires, Argentina, pp. 87-108.

Sedesol (Secretaría de Desarrollo Social) (2010), Diagnóstico sobre la situación de vulnerabilidad de la población de 70 años y más, Secretaría de Desarrollo Social, Ciudad de México, México.

Sen, Amartya (2000), Desarrollo y Libertad, Editorial Planeta, Ciudad de México, México.

Sojo, Ana (2004), Vulnerabilidad social y politicas públicas, Naciones Unidas-Comisión Económica para América Latina y el Caribe, Ciudad de México, México.

Székely, Miguel (2005), The economics of poverty, inequality and wealth accumulation in Mexico, MacMillan, Londres, Inglaterra.

Valdez, Elba Abril, Rosario Román y María José Cubillas (2005), “Análisis de la oferta de servicios para adultos mayores y sus necesidades manifiestas", Psicología y Salud, 15 (1), Instituto de Investigaciones Psicológicas de la Universidad Veracruzana, Veracruz, México, pp. 127-133. 
Villa, Miguel y Jorge Rodríguez (2002), Vulnerabilidad sociodemográfica: viejos y nuevos riesgos para comunidades, hogares y personas, Comisión Económica para América Latina y el Caribe, Brasilia, Brasil.

Ziccardi, Alicia (2000), "Las ciudades y la cuestión social", Revista de la escuela de economía y negocios, núm. 4, Universidad Nacional de General San Martín, Buenos Aires, Argentina, pp. 71-112.

Recibido: 2 de febrero de 2017.

Corregido: 5 de junio de 2017. Aceptado: 1 de septiembre de 2017.

Lucio Flores-Payan. Doctor en ciencias económico administrativas por la Universidad de Guadalajara, México. Actualmente es profesorinvestigador del Departamento de Políticas Públicas del Centro Universitario de Ciencias Económico Administrativas de la Universidad de Guadalajara. Es miembro del Sistema Nacional de Investigadores, nivel I. su línea de investigación es el análisis de políticas públicas mediante metodologías alternativas. Entre sus últimas publicaciones destacan: "Uso de conjuntos difusos para cuantificar el potencial de cuerpos académicos desde la conformación de redes científicas de colaboración”, Investigación y Ciencia, 24 (68), Universidad Autónoma de Aguascalientes, Aguascalientes, México, pp. 70-77 (2016); "Aplicación de conjuntos difusos para crear índices multidimensionales. Una aproximación para cuantificar la calidad del empleo", Acta Universitaria, Guanajuato, México, pp. 65-82 (2017).

Iván Alejandro Salas-Durazo. Es doctor en ciencias económico administrativas por la Universidad de Guadalajara, México. Actualmente es profesor-investigador del Departamento de Políticas Públicas del Centro Universitario de Ciencias Económico Administrativas de la Universidad de Guadalajara. Es miembro del Sistema Nacional de Investigadores, nivel I. Su línea de investigación actual es trayectorias laborales y educación superior. Entre sus últimas publicaciones destacan: "Aplicación de conjuntos difusos para crear índices multidimensionales. Una aproximación para cuantificar la calidad del empleo", Acta Universitaria, Guanajuato, México, pp. 65-82 (2017); "Análisis de las condiciones laborales de los estudiantes de dos carreras de una universidad pública mexicana. Una aproximación basada en conjuntos de lógica difusa", Revista Iberoamericana de Educación Superior, Universidad Nacional Autónoma de México, Ciudad de México, México, pp. 21-41 
(2016); "Modelado de conjuntos difusos para cuantificar la calidad del empleo. Una aproximación conceptual", Revista Ciencia y Universidad, Universidad Autónoma de Sinaloa, Culiacán, México, pp. 7-36 (2016). 